\title{
PREVALÊNCIA DE SOBREPESO E OBESIDADE EM CRIANÇAS MATRICULADAS EM UMA CRECHE DE BRASÍLIA-DF
}

\author{
PREVALENCE OF OVERWEIGHT AND OBESITY IN CHILDREN \\ ENROLLED IN A NURSERY OF BRASILIA-DF
}

\author{
Cláudia Ribeiro Borges Silva ${ }^{1}$, Andréia Araújo Lima Torres ${ }^{2}$
}

\begin{abstract}
${ }^{1}$ Nutricionista, Supervisora do Estágio do curso de Nutrição da Faculdade Alvorada, Brasília - DF.

${ }^{2}$ Nutricionista, Mestre, Professora do Departamento de Nutrição da Faculdade JK, Brasília - DF.
\end{abstract}

\section{Resumo}

A presente pesquisa trata-se de um estudo transversal com o objetivo de verificar a prevalência de sobrepeso e obesidade em crianças de 4 a 72 meses de idade, matriculadas em uma creche de Sobradinho-DF. Foram coletados dos arquivos da instituição dados como: peso, altura, sexo e data de nascimento de 53 crianças matriculadas na creche. Após a inserção destes dados no software WHO Anthro e WHO Anthroplus, as mesmas foram classificadas segundo os pontos de corte da tabela do Sistema de Vigilância alimentar e nutricional de 2008. O estado nutricional foi definido seguindo o índice de Massa Corporal - IMC para a idade sendo constatado que 3,78\% das crianças apresentaram baixo IMC, 13,20\% apresentaram sobrepeso e 3,78\% apresentaram obesidade. Conclui-se que, seguindo uma tendência mundial, tanto de países desenvolvidos como em desenvolvimento, o aumento na prevalência de sobrepeso e obesidade é alarmante. Porém, foi observado que obesidade e desnutrição coexistem devido à transição nutricional e as diferenças econômicas regionais existentes no Brasil.

Palavras Chave: Creche, Sobrepeso, Obesidade, Perfil nutricional, Índice de Massa Corporal.

\begin{abstract}
The present research it is a cross-sectional study aimed at verifying the prevalence of overweight and obesity in children aged 7 to 72 months of age, enrolled in a nursery Sobradinho-DF. We collected the data files of the institution as weight, height, sex and date of birth of 53 children enrolled in day care. After insertion of data in the WHO Anthro software and WHO Anthroplus, they were classified according to cutoff points table Surveillance System Food and Nutrition 2008. Nutritional status was defined following the Body Mass Index - BMI for age and found that $3.78 \%$ of children had low BMI, $13.20 \%$ were overweight and $3.78 \%$ were obese. We conclude that, following a worldwide trend, both developed and developing, the increase in prevalence of overweight and obesity is alarming. However, it was observed that malnutrition and obesity coexist due to the nutritional transition and regional economic disparities
\end{abstract}


Keywords: Creche, Overweight, obesity, nutritional profile, body mass index.

\section{Introdução}

As prevalências de sobrepeso e obesidade em populações adultas vêm crescendo não só em países desenvolvidos, mas também nos países em desenvolvimento (COSTA; CINTRA; FISBERG, 2006). No Brasil, verifica-se nas últimas décadas um processo de transição nutricional, constatando-se que entre os anos 1974/75 e 1989, houve uma redução da prevalência da desnutrição infantil (de 19,8\% para 7,6\%) e um aumento na prevalência de obesidade em adultos (de 5,7\% para 9,6\%) (OLIVEIRA; FISBERG, 2003). Em um estudo realizado em Recife foi mostrado que o sobrepeso e a obesidade já atingem 35\% dos escolares avaliados (BALABAN; SILVA, 2001).

Atentando-se a este fato, no início dos anos noventa a Organização Mundial de Saúde alertou sobre o aumento preocupante do excesso de gordura corpórea também nos jovens, após estimar que 18 milhões de crianças em todo o mundo, menores de 5 anos, apresentaram sobrepeso ou obesidade. E este aumento na prevalência de sobrepeso e obesidade, é alarmante e trazem impactos econômicos globais não só no presente, mas também já se pensando no que esses futuros adultos obesos poderão causar (SOARES; PETROSKI, 2003).

Dados disponíveis a esse respeito foram obtidos no Estudo Nacional da Despesa Familiar (ENDEF), realizado em 1974-75; na Pesquisa Nacional sobre Saúde e Nutrição (PNSN), realizado de junho a setembro de 1989; e na Pesquisa sobre Padrões de Vida (PPV), realizada em 1996-97 somente nas regiões Sudeste e Nordeste do país (COSTA; CINTRA; FISBERG, 2006). Na PNSN observou-se que a prevalência de obesidade em crianças e adolescentes era de 7,8\% e 7,6\%, respectivamente, sendo que entre as crianças menores de 10 anos era de $2,5 \%$ e $8 \%$ nas famílias de menor e maior renda respectivamente, vem aumentando consideravelmente fazendo com que a desnutrição e a obesidade coexistam (INAN,1991).

A avaliação do estado nutricional consiste em utilizar determinados procedimentos diagnósticos, possibilitando precisar a magnitude, o comportamento e os determinantes dos agravos nutricionais, permitindo a identificação de grupos de riscos e intervenções não apenas quanto aos efeitos, mas também ao das causas (RIBAS; PHILIPPI; ZORZATTO, 1999). Assim, o conhecimento e o acompanhamento da situação nutricional constituem instrumento essencial para a aferição das condições de saúde da população infantil, além de oferecer medidas objetivas das condições de vida da população em geral (FISBERG; MARCHIONI; CARDOSO, 2004).

No Brasil as instituições especializadas no atendimento de crianças, surgiram no século XIX, onde atendiam principalmente os filhos de mães solteiras de baixa renda. Posteriormente com a saída da mulher para o mercado de trabalho, surgiu um novo seguimento de creches, com a proposta de atender as necessidades das mães que trabalhavam na indústria. Então nas décadas de 70 e 80, houve uma intensificação da mobilização da sociedade civil no sentido de buscar a expansão do número de creches, e a partir disto as creches passaram a ser vistas como fonte de renda para seus funcionários e um direito das mães que trabalhavam fora (BOGUS, 2007). Assim, as creches constituem-se em um local de fácil acesso para se dar continuidade à avaliação nutricional dos pré escolares brasileiros. 
Este trabalho objetiva, portanto, verificar a prevalência de sobrepeso e obesidade entre as crianças de faixa etária que varia entre 4 meses de vida e seis anos de idade, em uma creche particular situada na cidade satélite de Sobradinho - DF.

\section{Metodologia}

Foi realizada uma pesquisa qualitativa utilizando instrumentos de coleta e análise de dados em um estudo transversal durante o segundo semestre de 2009, a fim de verificar a prevalência de sobrepeso e obesidade das 74 crianças matriculadas na creche com idades que variavam entre 4 a 72 meses. A avaliação nutricional das crianças matriculadas é realizada pela nutricionista da instituição a cada 6 meses; todas as informações como: peso, altura, data de nascimento, sexo, número de crianças matriculadas, foram extraídas dos bancos de dados fornecidos pela instituição.

As medidas de peso, sexo, altura e a data de nascimento foram inseridos no software WHO Antho versão de abril de 2009 para as crianças de faixa etária entre $4 \mathrm{e}$ 60 meses de idade, e WHO AnthroPlus versão de 2007 para aquelas compreendidas na faixa etária acima de 60 meses.

A partir dos dados fornecidos pelo software, foi obtida a classificação das crianças por z-escore no parâmetro IMC para idade. O software mensura o peso por estatura somente em crianças de ate 60 meses de idade, a partir daí este parâmetro não se aplica. Para a interpretação do escore $\mathrm{z}$ informado pelo software foi utilizada a tabela do Sistema de Vigilância Alimentar e Nutricional do Ministério da Saúde, que estabelece os pontos de corte para o diagnóstico nutricional.

Este projeto foi aprovado pelo Comitê de Ética em Pesquisa da Universidade de Brasília.

\section{Resultados}

Durante a avaliação nutricional realizada na instituição, as crianças ausentes neste dia não são avaliadas; desta forma, das 74 crianças matriculadas na creche somente 53 estavam presentes, destas 24 eram meninas e 29 eram meninos, representando $45 \%$ e $55 \%$ respectivamente da população estudada.

De acordo com o índice de massa corporal observou-se que 3,78\% apresentaram baixo IMC, 76,25\% apresentaram eutrofia, 13,20\% apresentaram sobrepeso e 3,78\% obesidade.

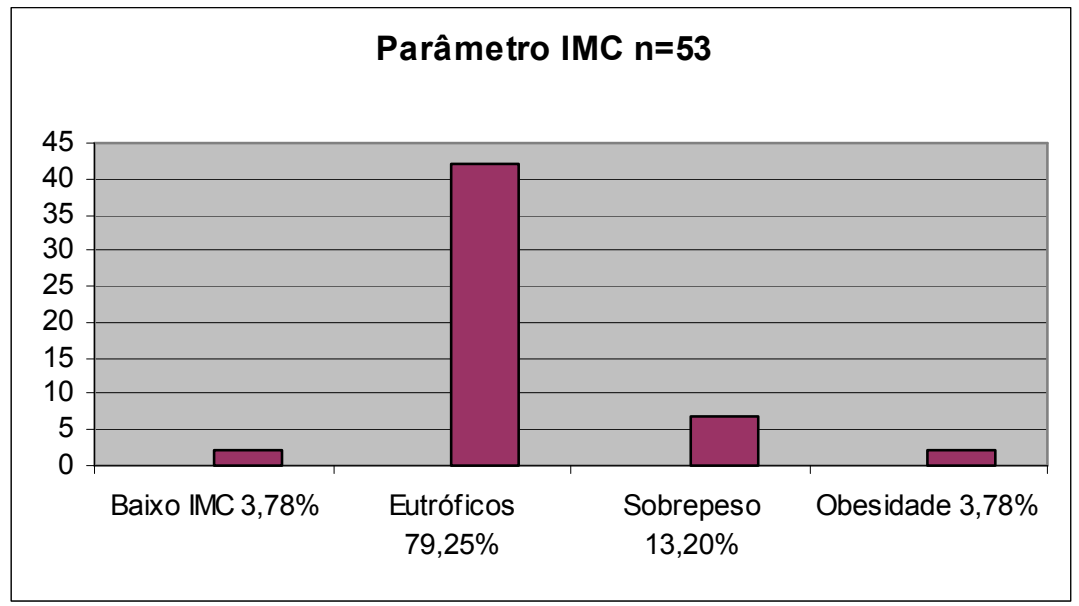

Figura 1: Representação gráfica da classificação por IMC por idade das crianças avaliadas, segundo as curvas da OMS. 


\section{Discussão}

No presente estudo o sobrepeso mostrou-se mais prevalente entre as crianças do sexo masculino $57 \%$, enquanto que entre as crianças do sexo feminino o percentual encontrado foi de $43 \%$, o que foi contrário aos resultados de estudos como de Balaban e Silva (2001), realizado em uma escola privada do Recife-PE, onde a prevalência de sobrepeso ocorreu em maior parte entre as crianças do sexo feminino, embora esta diferença estaticamente tenha sido insignificante. Utilizando os parâmetros da OMS Torres (2007), evidenciou em seu estudo, realizado em Brasília, uma alta prevalência de obesidade $(6 \%)$ dentre as crianças estudadas.

Diante da diversidade metodológica como índices e pontos de corte, a definição de obesidade torna-se complexa, entretanto, percebe-se maior utilização do IMC por idade em estudos mais recentes, tanto no Brasil como em outros países (LEÃO et al, 2003).

Durante Wokshop on chilhood obesity, o emprego do IMC foi recomendado pela IOTF - International Obesity Task Force para descrever a prevalência de obesidade em crianças e adolescentes de todo o mundo, pois, pode-se verificar a tendência das crianças de manterem o mesmo peso, acompanhando a evolução deste peso da infância ate a fase adulta, pela facilidade e baixo custo do emprego deste método. Assim, o IMC por idade revela-se um índice confiável e de fácil aplicação para a identificação de obesidade em nível populacional (LEÃO et al, 2003).

A distribuição de peso e IMC da OMS para avaliação sobrepeso e obesidade em pré-escolares privilegia a sensibilidade em detrimento da especificidade; o que pode ser uma decisão acertada diante da epidemia de excesso de peso que esta ocorrendo atualmente em nível mundial devido à transição nutricional (LEONE; BERTOLI; SCHOEPS, 2009).

Em 2001, Balaban relata em seu estudo que a obesidade foi mais freqüente em crianças do que em adolescentes de uma escola do Maranhão, e a prevalência de sobrepeso foi de $26,2 \%$ na população estudada, o mesmo concluiu que o sobrepeso consiste em um problema tão freqüente na amostra estudada quanto nos países desenvolvidos.

No presente estudo a prevalência de sobrepeso foi de $13,20 \%$ e 3,78\% tanto para a obesidade como para o baixo IMC o que é alarmante já que para uma população bem nutrida o esperado é $2,3 \%$ tanto para baixo peso como para excesso de peso, independentemente do referencial adotado (Torres, 2007; Rocha, 2007). O estudo confirma a conclusão do INAN (1991), segundo a qual em nosso país a obesidade e a desnutrição coexistem. Segundo Batista e Rissin (2003), o antagonismo de tendências temporais entre desnutrição e obesidade, definem uma das características marcantes do processo de transição nutricional do país.

\section{Conclusões}

Seguindo um padrão que se observa em países desenvolvidos e em desenvolvimento o aumento na prevalência de sobrepeso e obesidade estão em níveis muito acima do esperado, e aumentando de forma progressiva e rápida. Tal fato preocupa as autoridades de saúde em todo o mundo visto que as co-morbidades que afetam os obesos sobrecarregam o sistema de saúde e aumentam os gastos tanto para o 
governo quanto para o indivíduo, além de afetar negativamente a qualidade de vida do seu portador.

Daí a importância de estudos que verifiquem o estado nutricional ainda na infância, para que se possam traçar ações preventivas ao aparecimento da obesidade na fase adulta, podem-se monitorar o crescimento e desenvolvimento ideal da criança, detectando inadequações antes que as mesmas tornem-se um problema de saúde maior.

O resultado deste estudo foi encaminhado aos responsáveis pela creche para que ações corretivas sejam traçadas, tanto quanto à prevenção do excesso de peso quanto para a correção do baixo peso.

\section{Referências}

BALABAN, G.; SILVA, G.A.P. Prevalência de sobrepeso e obesidade em crianças e adolescentes de uma escola da rede privada de Recife. J. Pediatra. Rio Janeiro. v.77, n. 2, p. 96-100, 2001.

BATISTA FILHO, M.; RISSIN, A. A Transição nutricional no Brasil: tendências regionais e temporais. Cad. Saúde Pública. v.19, n. 1, p. 181-191, 2003.

BOGUS, C.M. Cuidados Oferecidos pelas Creches: percepções de mães e educadoras. Revista de Nutrição. São Paulo. v.20, n. 5, p.499-514, 2007.

COSTA, R.F. da; CINTRA, I. de P.; FISBERG, M. Prevalência de sobrepeso e obesidade em escolares da cidade de Santos, SP. Arq Bras Endocrinol Metab. v.50, n.1, p. 60-67, 2006.

FISBERG, R.M.; MARCHIONI, D.M.L; CARDOSO, M.R.A. Estado nutricional e fatores associados ao déficit de crescimento de crianças freqüentadoras de creches públicas do Município de São Paulo, Brasil. Cad. Saúde Pública. v. 20 n.3, 2004.

Instituto Nacional de Alimentação e Nutrição-BRASIL - MINISTÉRIO DA SAÚDE. Pesquisa Nacional de sobre Saúde e Nutrição. Condições nutricionais da população brasileira. Brasília, INAN, 1991.

LEAO, L.S.C. de S.; et al. Prevalência de obesidade em escolares de Salvador, Bahia. Arq Bras Endocrinol Metab. v.47, n. 2, p. 151-157, 2003.

LEONE, C.; BERTOLI, C.J.; SCHOEPS, D.O. Novas curvas de crescimento da Organização Mundial da Saúde: comparação com valores de crescimento de crianças pré-escolares das cidades de Taubaté e Santo André, São Paulo. Rev Paul Pediatr. v. 27, n.1, p.40-7, 2009.

OLIVEIRA, C.L.; FISBERG, M. Obesidade na infância e adolescência: uma verdadeira epidemia. Arq Bras Endocrinol Metab. v.47, n. 2, p. 107-108, 2003.

RIBAS, D.L.B.; et al. Saúde e estado nutricional infantil de uma população da região Centro-Oeste do Brasil. Rev. Saúde Pública. v.33, n. 4, p. 358-365, 1999. 
SOARES, LD; PETROSKI, EL. Prevalência, fatores etiológicos e tratamento da obesidade infantil. Revista Brasileira de Cineantropometria \& Desempenho Humano. v. 5, n. 1, p.63-74, 2003.

TORRES A.A.L.; FURUMOTO, R.A.V.; ALVES, E.D. Avaliação Antropométrica de pré-escolares - comparação entre os referenciais: NCHS 2000 e OMS 2005. Rev. Eletr. Enf. v. 9 n. 1, p.166-75, 2007.

VASCONCELOS, F.A.G. Avaliação Nutricional de Coletividades. $4^{\mathrm{a}}$ ed. Florianópolis: Ed. da UFSC, 2008. 